
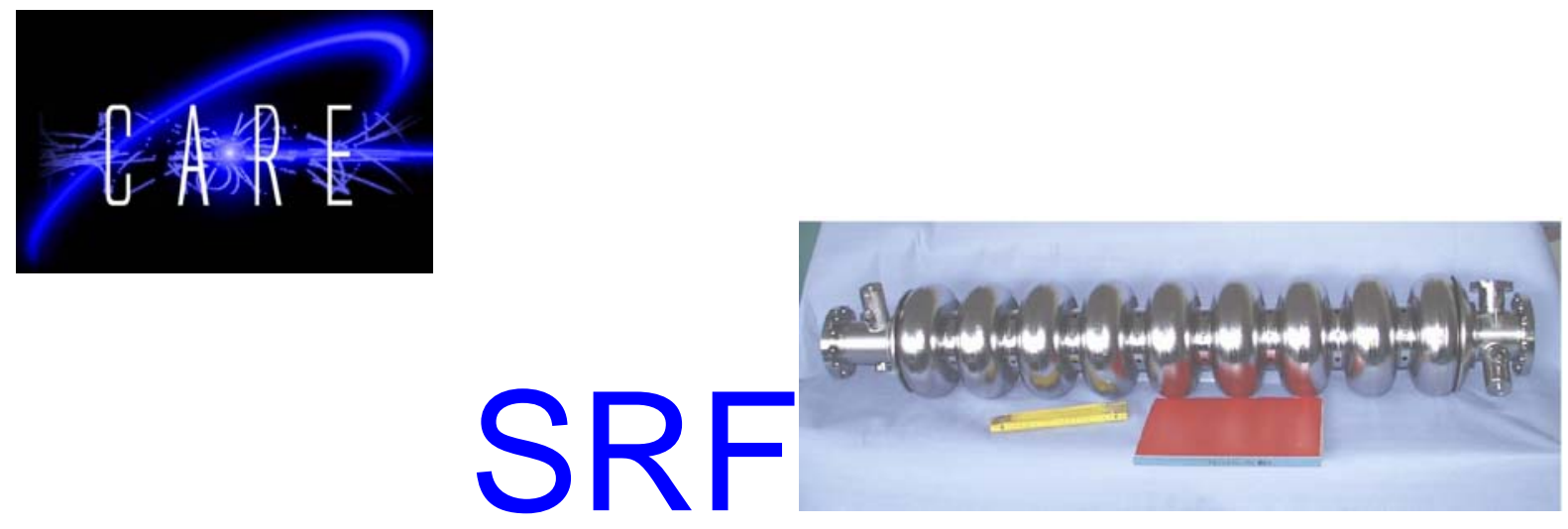

\title{
BEHAVIOUR OF GAS CONDITIONS DURING VACUUM ARC DISCHARGES USED FOR DEPOSITION OF THIN FILMS
}

\author{
P. Strzyzewski*, L. Catani ${ }^{\bullet}$, A. Cianchi ${ }^{\star}$, J. Langner*, J.Lorkiewicz $^{\star}$, R. Mirowski*, R. \\ Russo $^{\text {, M. Sadowski*, S. Tazzari }}{ }^{\text {a }}$ and J. Witkowski* \\ * The Andrzej Soltan Institute for Nuclear Studies, 05-400 Otwock/Swierk, Poland, e-mail: \\ p.strzyzewski@ipj.gov.pl@ Dipartamento di Fisica, Universita degli studi Roma "Tor \\ Vergata”, 00133 Roma, Italy $\bullet$ INFN-Roma2, Via della ricerca scientifica 1, 00133 Roma, \\ Italy Instituto di Cibernetica “E. Caianiello”, CNR, 80078 Pozzuoli, Italy
}

\begin{abstract}
The paper concerns an important problem which is connected with the inclusion of some impurities in the deposited metal film. It was found that appearance of contaminants in the film is induced mainly by water vapor remnants inside the vacuum chamber. The paper presents information on changes in the gas composition during and between arc-discharges, which is of primary importance for the selection of appropriate experimental conditions.
\end{abstract}

Contribution to the International Conference PLASMA-2005, Opole, Poland

Work supported by the European Community-Research Infrastructure Activity under the FP6 "Structuring the European Research Area" programme (CARE, contract number RII3-CT2003-506395). 


\title{
Behaviour Of Gas Conditions During Vacuum Arc Discharges Used For Deposition Of Thin Films
}

\author{
P. Strzyzewski*, L. Catani ${ }^{\bullet}$, A. Cianchi ${ }^{\bullet}$, J. Langner*, J.Lorkiewicz $^{\infty}$, R. \\ Mirowski*, R. Russo "M. Sadowski*, S. Tazzari and J. Witkowski* \\ * The Andrzej Soltan Institute for Nuclear Studies, 05-400 Otwock/Swierk, Poland, \\ e-mail: p.strzyzewski@ipj.gov.pl \\ "Dipartamento di Fisica, Universita degli studi Roma "Tor Vergata", 00133 Roma, Italy \\ - INFN-Roma2, Via della ricerca scientifica 1, 00133 Roma, Italy \\ "Instituto di Cibernetica "E. Caianiello", CNR, 80078 Pozzuoli, Italy
}

\begin{abstract}
The paper concerns an important problem which is connected with the inclusion of some impurities in the deposited metal film. It was found that appearance of contaminants in the film is induced mainly by water vapor remnants inside the vacuum chamber. The paper presents information on changes in the gas composition during and between arcdischarges, which is of primary importance for the selection of appropriate experimental conditions.

Keywords: Catodic Arc, Arc Deposition, Superconducting Film, RF Cavity, Quadropole Mass Spectrometer
\end{abstract}

PACS: $52.77 . \mathrm{Dq}, 52.80 . \mathrm{Mg}, 52.80 . \mathrm{Vp}$

\section{INTRODUCTION}

The vacuum arc, which is one of the oldest techniques used for the deposition of thin films, is now widely used for the Plasma Immersion Ion Implantation and Deposition (PIII\&D) in laboratory and industry [1]. Despite of high progress in this field observed during last three decades, involving e.g. magnetic filters for the elimination of microdroplets, some problems have not been resolved so far. One of them is believed to originate from the incorporation of impurities in a metal film. High adsorption of contaminants by thin films refers mainly to so-called getter materials, which can absorb impurities from the surrounding and dissolve them inside the deposited layer.

The paper concerns an important problem which is connected with the inclusion of some impurities in the deposited $\mathrm{Nb}$ film. It was found that the appearance of contaminants in the deposited film is induced mainly by water vapor remnants from the vacuum chamber. Particular attention is paid to a comparison of different gas conditions during arc discharges at high-vacuum conditions (at the background pressure in the range of $10^{-8}-10^{-7}$ $\mathrm{hPa}$ ) and at UHV experiments (at the background pressure within the range of $10^{-11}-10^{10} \mathrm{hPa}$ ).

\section{NIOBIUM AS A GETTER}

Getters are well known and often used in nowadays science and industry. The term "getters" was first introduce in 1882 by Thomas Edison's assistant Malignani. He developed a technique of the red-phosphorous deposition for increasing a lifetime of incandescent lamps. The red phosphorous reacts with water vapor and breaks the watertungsten cycle. This process is still in used in the lamp manufacturing. Moreover, getters are common in electron tubes and cathode ray tubes for television and computer monitor applications. The getter technology expanded also into a broad array of technologies and industries, e.g. in vacuum pumps for ultra-high vacuum (UHV) systems and particle accelerators, as well as in gas-purification systems for semiconductor processes and nanotechnology. Getters are used in these systems to pump away contaminants and/or keep the constant pressure inside. They work by the adsorption of gasses and chemical reactions. In a consequence, the layers of non-reactive oxides, carbides and nitrides can be created depending of adsorbed-gas type. 
Recently the vacuum arc technology was proposed as a possible alternative for depositing thin superconducting films of pure niobium on the inner surface of RF cavities for particle accelerators. Presence of the impurities usually deteriorates superconducting properties of niobium films. Since niobium is an excellent getter for vacuum residual gases a special technique of the thin film deposition must be applied. A new UHV cathodic-arc technique for the coating copper RF cavities was proposed in 2000 [2-3]. Results of residual gas conditions during deposition process, which are presented in the paper, show that numbers of contaminants are strongly reduced. Lowering the pressure to UHV standards can open way towards the production of ultra-pure metallic films. Preliminary studies, as performed at the IPJ in Swierk, Poland, in the collaboration with the Tor Vergata University team in Rome, Italy, have already shown that thin niobium films, which were deposited by arc-discharges under UHV conditions, demonstrate similar properties to the pure bulk niobium [4-5]. The UHV arc-deposited Nb-layers on sapphire substrates have been characterized by measuring their critical temperature $T_{c}$ and RRR, defined as the resistivity at room temperature divided by the resistivity at $10 \mathrm{~K}$. The critical temperature of the deposited material is in general very sensitive to impurities, e.g. very small amounts of oxygen in the $\mathrm{Nb}$-film can lower its $\mathrm{T}_{\mathrm{c}}$ value significantly. The $\mathrm{Nb} R R R$ is also very sensitive to impurities. Typical RRR values for $\mathrm{Nb}$-films deposited by sputtering at a room temperature have ranged from 20 to 50 .

\section{EXPERIMENTAL SET-UP}

For the coating of inner surfaces of RF accelerating structures, there were proposed various approaches. First one has been based on a linear (cylindrical) geometry of the arc source. A cylindrical cathode of the linear-arc can be placed along the cavity axis. Changing a position of a permanent magnet, which might be located inside of the tubular $\mathrm{Nb}$ cathode, one can drive the arc discharge along the symmetry axis - in order to obtain uniform coating of a single cavity or multi-cell structure. A scheme of such a system, which has been designed at IPJ, is shown in Fig.1.

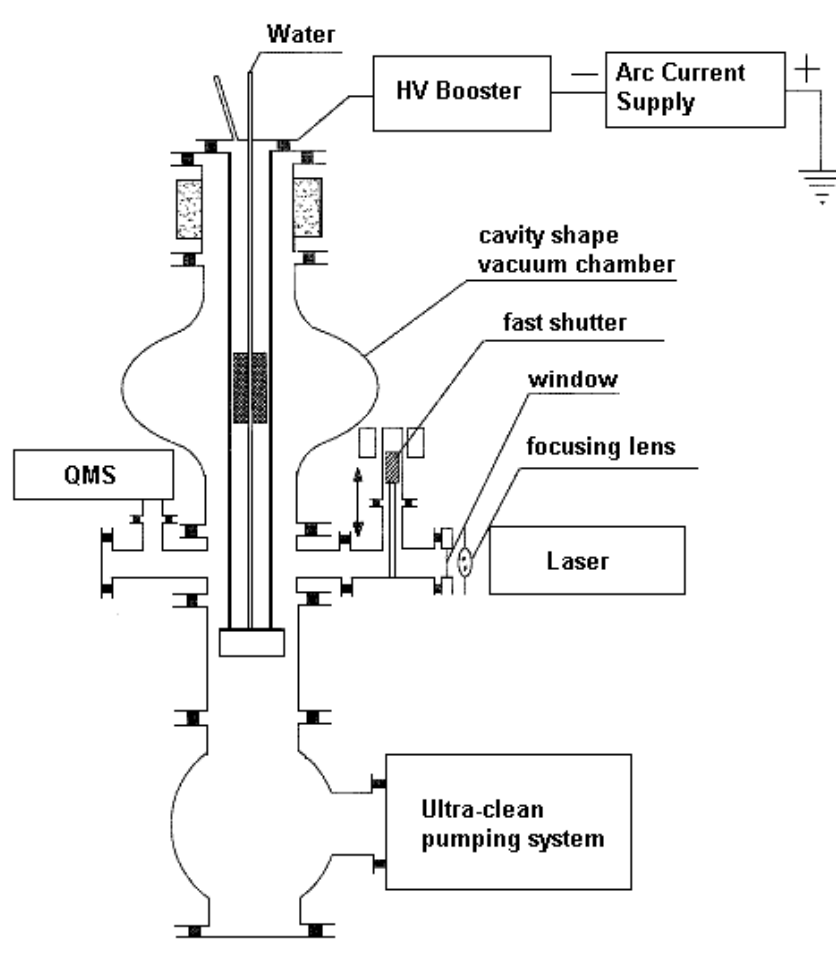

FIGURE 1. Schematic drawing of an UHV apparatus with the linear-arc source for single-cell cavity coating.

The facility is pumped down to $10^{-11} \mathrm{hPa}$ with an oil-free pumping system consisting of a two-stage piston pump as a fore-line of a turbo molecular pump. The low pressure is achieved only when all parts of the deposition system are designed and built in accordance with the UHV-technology requirements. In our case, all the vacuum chamber components and accessories, as well as all vacuum connections, have been manufactured using only high purity materials: stainless-steel, OFHC copper and high-quality ceramics (shielded from the arc). The cathode and all parts accessible to the arc have been made of pure $\mathrm{Nb}$ with $R R R \geq 250$.

The reliable triggering (ignition) of arc discharges is often a serious problem, even in the industrial arcbased devices. In HV systems, thin layers of gasses and impurities formed upon the surface of electrodes, are beneficial in that sense that they facilitate the starting of an arc discharge. Under UHV conditions the high-temperature baking of the vacuum chamber removes almost totally such layers. The described effects, as well as requirements that all other sources of impurities must be effectively eliminated, make the arc ignition more difficult. After testing many known triggering methods from the point of view of the operational reliability and cleanness, we have finally decided to use a laser beam focused upon the cathode surface (through an appropriate vacuum-tight glass window). Under such conditions the arc discharge can be triggered extremely reliably without introducing any additional impurity whatsoever [6]. Our arc sources have been equipped with Nd:YAG lasers (50-100mJ, 10ns), and the mastering of the laser ignition technique has appeared to be decisive for improving properties of the deposited superconducting films. 


\section{COMPARISON OF HV AND UHV CONDITIONS}

The composition of residual gases before and during the coating process is monitored with a Residual Gas Analyzers (RGA). A Quadropole Mass Spectrometer, used in the experiment, seems to be a very useful tool for qualitative and quantitative analysis of residual gases at UHV conditions. Typical examples of mass-spectra, presenting gas conditions at pressures $10^{-8} \mathrm{hPa}$ (before baking) and $10^{-10} \mathrm{hPa}$ (after baking), are shown in Fig.2.
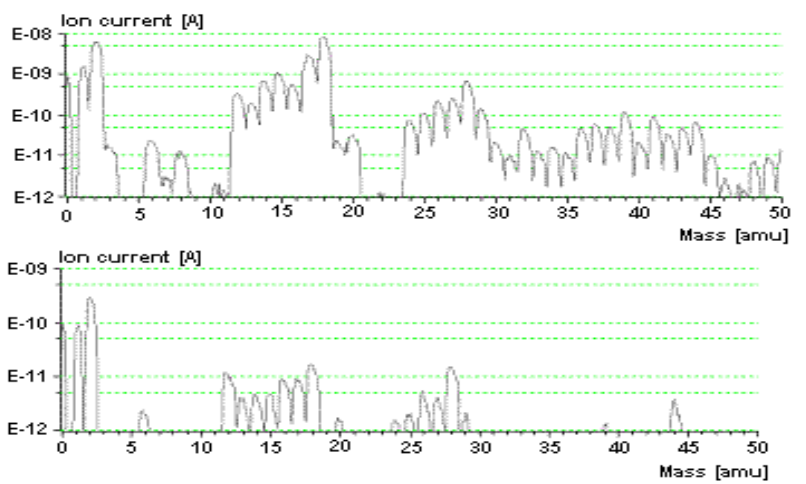

FIGURE 2. Typical RGA mass-spectra recorded before and after 24 -hour baking at $150{ }^{\circ} \mathrm{C}$

The presented mass-spectra demonstrate very well differences between the HV and UHV conditions. One can see that reaching UHV standards can cause the practical elimination of impurities from the vacuum chamber, i.e. water remnants (mass 18), nitrogen (mass 28) and $\mathrm{C}_{\mathrm{x}} \mathrm{H}_{\mathrm{y}}$ groups (associated with masses 38-43). Under such conditions hydrogen became the most dominant gas. Obtaining such "clean" conditions allow us to start the deposition process.

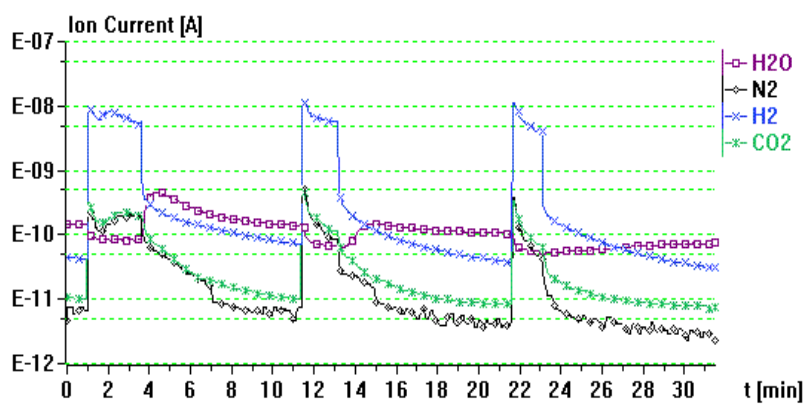

FIGURE 3a. Behavior of the partial pressure (in ion-current units) residual gases in $\mathrm{HV}$ conditions during the arc.

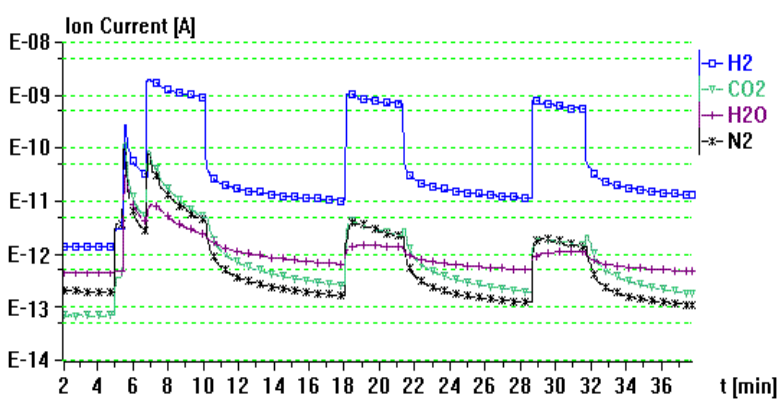

FIGURE 3b. Behavior of the partial pressure (in ion-current units) residual gases in UHV conditions during the arc.

Figs. $3 \mathrm{a}$ and $3 \mathrm{~b}$ show results of the time-resolved measurements of the selected ion masses at the HV and UHV conditions. Each of the diagrams presents three pulses of gas during the arc discharge, with some breaks between. Length of the discharge pulse was about 2-3 minutes. In both cases, immediately after the start of arc discharge, one could observe the out-gassing of gases from the cathode. The pressure increased up to $10^{-6}-10^{-7} \mathrm{hPa}$ when the arc discharge was started, and it remained almost stable (at the latter value) throughout the whole deposition process. The gas pressure rise during the arc discharge was found to be almost exclusively caused by hydrogen. Its partial pressure at the UHV condition is usually about 2-3 orders of magnitude higher than that of other gases. However, when the deposition is performed at HV, the level of hydrogen was only 1-2 orders of magnitude higher. After 40-60 seconds the partial pressure of out-gassed species, excluding water vapor, began to fall slightly. After the start of the arc at $\mathrm{HV}$ conditions some decrease in water vapor pressure was observed. It was probably caused by the formation of a fresh oxide-free niobium surface, which could absorb water vapor strongly. Such phenomena have also been observed during arcing at UHV conditions, where amount of water vapor is the smallest in comparison to $\mathrm{H}_{2}, \mathrm{CO}_{2}$ and $\mathrm{N}_{2}$. As soon as the arc extinguishes the hydrogen pressure drops about one order of magnitude, reaching its equilibrium pressure in presence of the niobium surface, and then starts slowly decreasing, pumped by the $200 \mathrm{l} / \mathrm{s}$ turbo-molecular pump. Water vapor becomes again the most dominant gas in HV conditions. Figure $3 \mathrm{~b}$ presents also 
behavior of gases when laser triggering pulse hit the cathode but arc discharge doesn't start. This situation is shown as a first short peak.

Figures $4 \mathrm{a}-4 \mathrm{~d}$ present compositions of residual gases in four different cases and they allow easy to compare the $\mathrm{HV}$ and UHV conditions. The measurements were performed before arc discharges (as the steady-state case) and about $30 \mathrm{~s}$ after the arc stopping. It is important to note that a percentage of water dropped from $80 \%$ at $\mathrm{HV}$ before the discharge - to about $3 \%$ at UHV after the discharge. In that case the residual gas (inside the vacuum chamber) was mainly hydrogen. Percentage of other gases could be neglected.

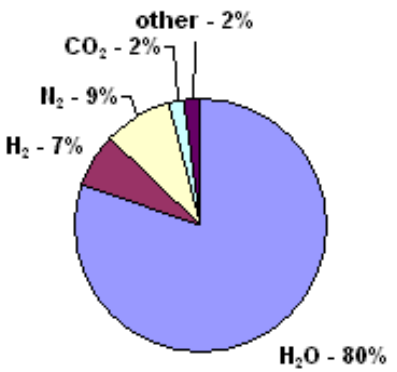

a

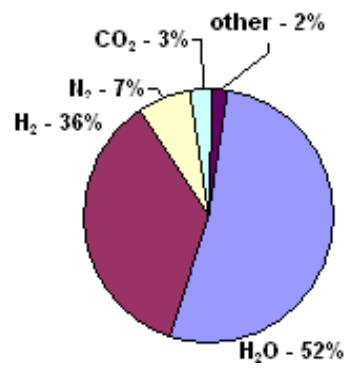

b

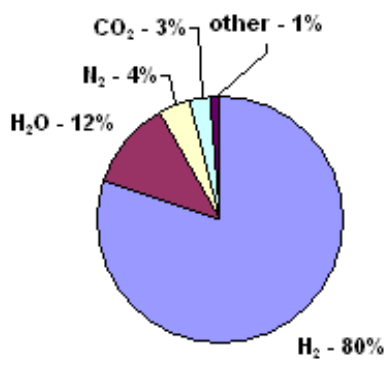

C

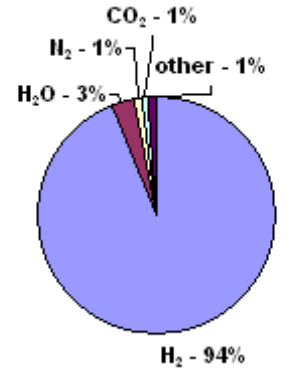

d

FIGURE 4. Residual gas compositions in vacuum chamber in: HV conditions ("a" - before arc discharge at $5 \times 10^{-8} \mathrm{hPa}$, "b" after arc discharge at $9 \times 10^{-7} \mathrm{hPa}$ ) and UHV conditions ("c" - before arc discharge at $6 \times 10^{-10} \mathrm{hPa}$, "d" - after arc discharge at $\left.9 \times 10^{-9} \mathrm{hPa}\right)$.

\section{SUMMARY}

According to our previous papers all impurities, which are present at HV conditions, can considerably influence quality of the deposited superconducting niobium films. This paper has clearly showed differences between the HV and UHV conditions. Performing depositions by means of vacuum arc discharges at the UHV conditions can guarantee a very low level of unwanted gases, mainly water vapor. This is especially important during dc- or pulsedtype arc-discharges, as well as after them, because it prevents oxidizing of the surface layers during the cooling down process.

\section{ACKNOWLEDGEMENT}

We acknowledge the support of the European Community-Research Infrastructure Activity under the FP6 "Structuring the European Research Area" programme (CARE, contract number RII3-CT-2003-506395).

\section{REFERENCES}

1. A. Anders, Vacuum 67, 2002, 673.

2. J. Langner, in TESLA Report 2000-15. Edit.: D. Proch, 2000 DESY.

3. R. Russo, et al., in Proc. X Workshop on RF superconductivity, Tsukuba 2001, KEK Proc. 2003-2, 4.

4. J. Langner, et al., Czech. J. Phys. 54, Suppl.C, 914 (2004).

5. R. Russo, et al., Supercond. Sci. Technol. 18, 2005, L41-L44.

6. J. Langner, at al., Proc. International Conference on Plasma Research and Applications PLASMA-2003, Warsaw, Poland, September 9-12, 2003. 